\title{
Smaller Explicit Superconcentrators
}

\author{
N. Alon and M. Capalbo
}

Abstract. Using a new recursive technique, we present an explicit construction of an infinite family of $N$-superconcentrators of density 44 . The most economical previously known explicit graphs of this type have density around 60 .

\section{Introduction}

For an integer $N$, an $N$-superconcentrator $\Gamma_{N}$ is a directed acyclic graph with a set $X$ of $N$ inputs (i.e., vertices with indegree 0 ) and a set $Y$ of $N$ outputs (i.e., vertices with outdegree 0 ), such that, for any subset $S$ of $X$, and any subset $T$ of $Y$ satisfying $|S|=|T|$, there are $|S|$ vertex-disjoint directed paths in $\Gamma_{N}$ from $S$ to $T$. Superconcentrators have many applications in computer science and in the study of communication networks, see, e.g., [Valiant 76], [Chung 78], [Pippenger 77], and their references. The explicit construction of sparse graphs of this type has been an extensively studied problem. Gabber and Galil [Gabber and Galil 79] presented the first explicit construction of $N$-superconcentrators with $O(N)$ edges (about $270 N$ edges). Since then, several researchers ([Alon and Milman 85], [Buck 86], [Jimbo and Maruoka 87], [Morgenstern 94], and [Alon et al. 87]) presented constructions of $N$-superconcentrators using fewer and fewer directed edges. The most economical construction before the one described in the present paper has been obtained from the technique presented in [Alon et al. 87], combined with the Ramanujan graphs constructed in [Lubotzky et al. 88], and yields a family of $N$-superconcentrators that have about $60 N$ edges. All of these

(C) A K Peters, Ltd.

I542-795I/04 $\$ 0.50$ per page 
constructions use the same recursive technique, and achieve improvements by using either better explicit expander constructions, or better analysis of known expander constructions. The best known lower bound for the number of edges of an $N$-superconcentrator is only $(5-o(1)) N$, proved by Lev and Valiant in [Lev and Valiant 83].

In this paper, we present a construction of a family of $N$-superconcentrators $\Gamma_{N}$ with only $44 N+O(1)$ edges. This is done by introducing a new recursive technique, and by combining it with appropriate extended double covers of Ramanujan expanders. In Section 2, we briefly describe the Ramanujan graphs needed here, and in Section 3, we give the construction of the graphs $\Gamma_{N}$. The proof of the main result, that each $\Gamma_{N}$ is indeed an $N$-superconcentrator, is given in Section 4.

Our notation is mostly standard. For a graph $G=(V(G), E(G))$, and for a set $S \subseteq V(G)$, let $\mathcal{N}_{G}(S)$ denote the set of vertices in $G$ that are adjacent in $G$ to at least one vertex in $S$. If $M$ is a matching in $G, v$ is a vertex of $G$, and $S$ is a subset of vertices of $G$, we say that $M$ covers $v$ if $v$ is incident with an edge in $M$. $M$ saturates $S$ if it covers every vertex of $S$.

For an undirected graph $\Lambda=(X, E)$, where $X=\left\{x_{1}, \ldots, x_{N}\right\}$ is a set of $N$ vertices, the extended double cover of $\Lambda$ is the bipartite graph $\Lambda^{\prime}$ with parts $Y=\left\{y_{1}, \ldots, y_{N}\right\}$ and $Z=\left\{z_{1}, \ldots, z_{N}\right\}$, where $y_{\iota} z_{\iota^{\prime}}$ is an edge in $\Lambda^{\prime}$ if and only if either $\iota=\iota^{\prime}$, or $x_{\iota} x_{\iota^{\prime}}$ is an edge in $\Lambda$. Thus, if $\Lambda$ is loopless and $k$-regular, then its extended double cover $\Lambda^{\prime}$ is $k+1$-regular.

A graph is called Ramanujan if it is $d$-regular for some $d>2$, and the absolute value of each eigenvalue of its adjacency matrix besides the largest one, is at most $2 \sqrt{d-1}$.

For a group $G$ and a subset $\Sigma$ of $G$, where $\pi \in \Sigma$ if and only if $\pi^{-1} \in \Sigma$, the Cayley graph $\Lambda^{\prime}$ on $G$ with respect to $\Sigma$ is the $|\Sigma|$-regular graph whose vertex-set is $G$, and whose edge-set is $\{\{\nu, \pi \nu\} \mid \nu \in G$ and $\pi \in \Sigma\}$. The elements $\pi \in \Sigma$ are the generators of $\Lambda^{\prime}$, and $\Sigma$ is the set of generators of $\Lambda^{\prime}$. If $\Sigma$ generates $G$, then $\Lambda^{\prime}$ is connected; otherwise, the components of $\Lambda^{\prime}$ are the right cosets of the subgroup $S$ of $G$ generated by $\Sigma$ (i.e., the sets of the form $\{s g \mid s \in S\}$ ). If $H$ is a subgroup of $G$, the Schreier graph $(G ; G / H ; \Sigma)$ is the graph whose vertices are the left cosets of $H$, where $s H$ and $s^{\prime} H$ are adjacent if there exists a $\pi \in \Sigma$ such that $\pi s H=s^{\prime} H$.

\section{An Auxiliary Construction}

We need an infinite series of explicit 9-regular Ramanujan graphs $\Lambda_{1}, \ldots, \Lambda_{l}, \Lambda_{l+1}, \ldots$ such that $\Lambda_{l+1}$ has exactly twice as many vertices as $\Lambda_{l}$. The construction in 
[Morgenstern 94] gives us 9-regular Ramanujan graphs, but not in the sizes that we need. Thus, we present the following modification shown to us by A. Lubotzky.

Theorem 2.I. (A. Lubotzky [Lubotzky 02]) There exists an explicit family of 9regular Ramanujan graphs on $k \times 2^{l-1}$ vertices, where $k=64 \times 65 \times 63=$ 262,080 , and $l=0,1,2, \ldots$.

Proof. We first construct an explicit family $\mathcal{H}^{\prime}$ of 9-regular Ramanujan graphs on $k \times 2^{18 l-18}$ vertices, for each integer $l$. Then we construct $\mathcal{H}$ from $\mathcal{H}^{\prime}$.

To construct $\mathcal{H}^{\prime}$, let $g(x)$ be an irreducible polynomial of degree 2 in $F_{8}[x]$, where $F_{8}$ denotes the field on 8 elements. For each nonnegative integer $l$, the graph $\Lambda_{l}^{\prime} \in \mathcal{H}^{\prime}$ is the Cayley graph on $H_{l}=P G L_{2}\left(K_{l}\right)$ where $K_{l}=$ $F_{8}[x] / g^{l}(x) F_{8}[x]$ with respect to the set $\Sigma_{l}$ of generators, specified below. The number of vertices of this graph is $k \times 2^{18 l-18}$, where $k$ is as above. To see this, note that $K_{1}$ is the finite field with 64 elements, and there are precisely $\left(64^{2}-1\right)\left(64^{2}-64\right)$ two-by-two invertible matrices over it. This is, therefore, the number of ways to choose the entries of an invertible two-by-two matrix over $K_{l}$ modulo $g(x)$. Knowing these, there are $8^{(2 l-2) 4}$ ways to choose the actual entries, and this way, we obtain all the invertible matrices over $K_{l}$. Since $K_{l}$ contains $8^{2 l}-8^{2 l-2}$ elements which are not divisible by $g(x)$, this implies that the cardinality of $P G L_{2}\left(K_{l}\right)$ is $\left(64^{2}-1\right)\left(64^{2}-64\right) 8^{(2 l-2) 4} /\left(8^{2 l}-8^{2 l-2}\right)=k 2^{18 l-18}$.

To specify $\Sigma_{l}$, first fix a $\gamma$ in $F_{8}$ such that the resulting polynomial $q(x)=$ $x^{2}+x+\gamma$ is irreducible in $F_{8}[x]$. Next, let $\beta_{l}$ be a root of $q(x)$ in $F_{8}[x] / g^{l}(x) F_{8}[x]$ $=K_{l}$. The proof of existence of such a root is given in the following proposition.

Proposition 2.2. Let $q(x)=x^{2}+x+\gamma$ be an irreducible polynomial in $F_{8}[x]$, and let $g(x)$ be any other irreducible polynomial of degree 2 in $F_{8}[x]$. Then for each positive integer $l$, there is a root $\beta_{l}$ of $q(x)$ in the ring $K_{l}=F_{8}[x] / g^{l}(x) F_{8}[x]$. Furthermore, we can find such a $\beta_{l}$ efficiently.

Proof. Observe, first, that as $K_{1}=F_{8}[x] / g(x) F_{8}[x]$ is the unique finite field with $8^{2}=64$ elements, it contains the roots of every irreducible polynomial of degree 2 over $F_{8}$, and hence, contains a root $\beta_{1}$ of $q(x)$ in the field $K_{1}$.

To complete the proof of Proposition 2.2, apply induction on $l$. Let $l$ be any positive integer. We assume that there exists a root $\beta_{l}$ of $q(x)$ in $K_{l}$, or equivalently, there exists a $\beta_{l} \in F_{8}[x]$ such that $q\left(\beta_{l}\right) \equiv 0 \bmod g^{l}(x)$. Now let $\beta_{l+1}$ be the element in $F_{8}[x]$ such that $\beta_{l+1}=\beta_{l}+r(x) g^{l}(x)$, where $r(x)=r$ is 
a polynomial of degree at most 1 in $F_{8}[x]$ to be determined later. Then

$$
q\left(\beta_{l+1}\right)=q\left(\beta_{l}\right)+r(x) g^{l}(x)+r^{2}(x) g^{2 l}(x) .
$$

But if $l$ is positive, then $2 l \geq l+1$. Also, as $q\left(\beta_{l}\right) \equiv 0 \bmod g^{l}(x)$, it follows that $q\left(\beta_{l}\right) \equiv \tilde{r}(x) g^{l}(x) \bmod g^{l+1}(x)$ for some $\tilde{r}(x)$ in $F_{8}[x]$ of degree at most 1 . Set $r(x)=\tilde{r}(x)$ to conclude that $q\left(\beta_{l+1}\right) \equiv 0 \bmod g^{l+1}(x)$. Obviously, this proof yields an efficient algorithm as well.

Let $\Sigma_{l}$ be the following 9-element subset of $H_{l}$.

$$
\Sigma_{l}=\left\{\left(\begin{array}{cc}
1 & \varepsilon+\delta \beta_{l} \\
\left(\varepsilon+\delta \beta_{l}+\delta\right) x & 1
\end{array}\right) \mid \delta, \varepsilon \in F_{8} ; \quad \varepsilon^{2}+\varepsilon \delta+\delta^{2} \gamma=1\right\} .
$$

It is easy to check that $\Sigma_{l}$ has 9 elements and that each element of it is of order 2 in $P G L_{2}\left(K_{l}\right)$. This completes the description of all graphs $\Lambda_{l}^{\prime} \in \mathcal{H}^{\prime}$. By the proof in [Morgenstern 94], each $\Lambda_{l} \in \mathcal{H}^{\prime}$ is Ramanujan.

To construct $\mathcal{H}$ from $\mathcal{H}^{\prime}$, we do the following. Let $\varphi$ be the following mapping

$$
\pi=\left(\begin{array}{ll}
a_{11} & a_{12} \\
a_{21} & a_{22}
\end{array}\right) \mapsto\left(\begin{array}{ll}
a_{11}^{\prime} & a_{12}^{\prime} \\
a_{21}^{\prime} & a_{22}^{\prime}
\end{array}\right)
$$

from $H_{l+1}$ to $H_{l}$, where $a_{i j}^{\prime}=a_{i j} \bmod g^{l}(x)$ for each $i, j \in\{1,2\}$. Then $\varphi$ is a surjective mapping and a group homomorphism, and the kernel of $\varphi$ is $H^{\prime}$, where

$$
H^{\prime}=\left\{\left(\begin{array}{cc}
1+g^{l}(x) s(x) & g^{l}(x) r(x) \\
t(x) g^{l}(x) & 1
\end{array}\right) \mid r(x), s(x), t(x) \in F_{8}[x] / g(x) F_{8}[x]\right\} .
$$

$H^{\prime}$ is a group, and is isomorphic to $F_{2}^{18}$, where addition in $F_{2}^{18}$ corresponds to multiplication in $H^{\prime}$. Thus, for each positive integer $c \leq 18$, there is a subgroup $H_{c}^{\prime}$ of $H$ with $2^{c}$ elements. Also, if $\pi$ and $\pi^{\prime}$ are distinct elements in $\Sigma_{l+1}$, then $\varphi(\pi) \neq \varphi\left(\pi^{\prime}\right)$, as shown in the following claim.

Claim 2.3. Let $\Sigma_{l+1}$ and $\varphi$ be as above. If $\pi$ and $\pi^{\prime}$ are distinct elements in $\Sigma_{l+1}$, then $\varphi(\pi) \neq \varphi\left(\pi^{\prime}\right)$.

Proof. For any two polynomials $s(x), t(x) \in F_{8}[x]$, say that $t(x)$ is a multiple of $s(x)$ if $t(x)=r(x) s(x)$, for some polynomial $r(x) \in F_{8}[x]$. Let $\pi$ and $\pi^{\prime}$ be two distinct elements in $\Sigma_{l+1}$ and suppose that $\varphi(\pi)=\varphi\left(\pi^{\prime}\right)$. Then for some $\delta, \epsilon \in F_{8}$, where at least one is nonzero, $\epsilon+\delta \beta_{l+1}$ is a multiple of $g^{l}(x)$. We may assume that $\delta$ is nonzero, and by dividing, if needed, that $\delta=1$. Hence, 
$\beta_{l+1}+\epsilon$ is a multiple of $g^{l}(x)$. Now let us write $q(x)=(x+\epsilon) x+r(x)$, where $r(x)$ is a polynomial in $F_{8}[x]$ that has degree at most 1 . Because $q$ is irreducible in $F_{8}[x], r(x)$ is not a multiple of $x+\epsilon$. However, then $r\left(\beta_{l+1}\right)$ is a multiple of $g^{l}(x)$ because $q\left(\beta_{l+1}\right)$ is a multiple of $g^{l}(x)$ (by the definition of $\beta_{l+1}$ ) and so is $\beta_{l+1}+\epsilon$. But since $r(x)$ is not a multiple of $x+\epsilon$, this implies that $\epsilon^{\prime}$ is a multiple of $g^{l}(x)$ for some nonzero $\epsilon^{\prime} \in F_{8}$, which is impossible.

By the above discussion, it follows that for each positive integer $c \leq 18$, the Schreier graph $\left(H_{l+1} ; H_{l+1} / H_{c}^{\prime} ; \Sigma_{l+1}\right)$ is 9-regular, Ramanujan, and has $k \times 2^{18 l-c}$ vertices. The construction of a 9-regular Ramanujan graph $\Lambda_{l}$ on $k \times 2^{l}$ vertices for each $l \geq 0$ follows.

\section{Explicit Superconcentrators}

Here, we construct an infinite family of $N$-superconcentrators with $44 N+O(1)$ edges for each $N$ of the form $k \times 2^{l}$, and each nonnegative integer $l$. Let $\Gamma_{k}$ be any explicit $k$-superconcentrator from [Alon et al. 87] or [Jimbo and Maruoka 87] (since $k=O(1)$, the exact size of $\Gamma_{k}$ does not really matter). We next present the construction of $\Gamma_{N}$ for each $N$ of the form $k \times 2^{l}$, and each positive integer $l$.

\section{I. The Construction of $\Gamma_{N}$}

Let $X$ and $Y$ be disjoint sets of $N$ vertices each. The input and output sets of $\Gamma_{N}$ are $X$ and $Y$, respectively. Let $X^{\prime}=\left\{x_{1}^{\prime}, \ldots, x_{N}^{\prime}\right\}$ and $Y^{\prime}=\left\{y_{1}^{\prime}, \ldots, y_{N}^{\prime}\right\}$ be disjoint sets of $N$ vertices each.

The edges in $\Gamma_{N}$ between $X$ and $X^{\prime}$ form the extended double cover $\Lambda_{X}$ of a 9-regular Ramanujan graph (explicitly constructed in Theorem 2.1), where each edge is directed from $X$ to $X^{\prime}$. Similarly, the edges in $\Gamma_{N}$ from $Y^{\prime}$ to $Y$ form the extended double cover $\Lambda_{Y}$ of a 9-regular Ramanujan graph, where each edge is directed from $Y^{\prime}$ to $Y$. In addition, for each $\iota \in\{1, . ., N / 2\}$, the $\operatorname{arcs}\left(x_{\iota+N / 2}^{\prime}, y_{\iota}^{\prime}\right)$, $\left(x_{\iota+N / 2}^{\prime}, x_{\iota}^{\prime}\right),\left(x_{\iota}^{\prime}, y_{\iota+N / 2}^{\prime}\right)$, and $\left(y_{\iota}^{\prime}, y_{\iota+N / 2}^{\prime}\right)$ are in $\Gamma_{N}$.

Put $X^{\prime \prime}=\left\{x_{\iota}^{\prime} \in X^{\prime} \mid \iota \in\{1, \ldots, N / 2\}\right\}$, and $Y^{\prime \prime}=\left\{y_{\iota}^{\prime} \in Y^{\prime} \mid \iota \in\{1, \ldots, N / 2\}\right\}$. The remaining edges of $\Gamma_{N}$ form a digraph with input set $X^{\prime \prime}$ and output set $Y^{\prime \prime}$, which is isomorphic to $\Gamma_{N / 2}$, and is vertex-disjoint from $X, Y, Y^{\prime} \backslash Y^{\prime \prime}$, and $X^{\prime} \backslash X^{\prime \prime}$.

The rest of this paper is devoted to proving the following theorem.

Theorem 3.I. $\Gamma_{N}$ has $44 N+O(1)$ edges, and is an $N$-superconcentrator, with input set $X$ and output set $Y$. 


\section{The Proof of Theorem 3.I}

Proof. By construction, the number of edges $f(N)$ of $\Gamma_{N}$ satisfies $f(N)=(2 \cdot 10+$ 2) $N+f(N / 2)$, implying the claim about the number of edges of $\Gamma_{N}$.

To prove the main part of Theorem 3.1, we use induction; that is, we assume that $\Gamma_{N / 2}$ is an $N / 2$-superconcentrator, and show that $\Gamma_{N}$ is an $N$-superconcentrator. Let $S$ be any subset of $X$, and let $T$ be any subset of $Y$ such that $|S|=|T|$. We first show that:

(A) there is a set $\mathcal{P}$ of vertex-disjoint directed paths in $\Gamma_{N}$ in $X \cup X^{\prime} \cup Y \cup Y^{\prime}$ such that

(i) each vertex in $S \cup T$ is an endpoint of exactly one $P \in \mathcal{P}$, and

(ii) each $P \in \mathcal{P}$ is either (a) from $S$ to $T$, (b) from $S$ to $X^{\prime \prime}$, or (c) from $Y^{\prime \prime}$ to $T$.

Then (A), together with our inductive hypothesis that $\Gamma_{N / 2}$ is an $N / 2$-superconcentrator, implies that $\Gamma_{N}$ is an $N$-superconcentrator.

We next show the existence of such a $\mathcal{P}$. We make the following claim.

Lemma 4.I. Let $S$ be any subset of $X$, and let $T$ be any subset of $Y$ where $|S|=|T|$. Suppose there exist matchings $M_{S}^{*} \subset \Lambda_{X}$ and $M_{T}^{*} \subset \Lambda_{Y}$ such that both $M_{S}^{*}$ and $M_{T}^{*}$ have $|S|=|T|$ edges, and $M_{S}^{*}$ and $M_{T}^{*}$ satisfy properties (1) and (2), stated below.

(1) $M_{S}^{*}$ saturates $S$, and $M_{T}^{*}$ saturates $T$.

(2) Let $\iota$ be an arbitrary integer in $\{1,2, \ldots, N / 2\}$. Then if $M_{S}^{*}$ covers both $x_{\iota}^{\prime}$ and $x_{\iota+N / 2}^{\prime}$, then $M_{T}^{*}$ covers at least one vertex of $\left\{y_{\iota}^{\prime}, y_{\iota+N / 2}^{\prime}\right\}$. Similarly, if $M_{T}^{*}$ covers both $y_{\iota}^{\prime}$ and $y_{\iota+N / 2}^{\prime}$, then $M_{S}^{*}$ covers at least one of $\left\{x_{\iota}^{\prime}, x_{\iota+N / 2}^{\prime}\right\}$.

Then there exists a collection $\mathcal{P}$ of vertex-disjoint paths in $\Gamma_{N}$ in $X \cup X^{\prime} \cup Y^{\prime} \cup Y$ that satisfies $(\mathbf{A})$.

Proof. Let $X_{S}^{\prime}$ be the set of vertices $x^{\prime} \in X^{\prime}$ that are covered by an edge in $M_{S}^{*}$, and let $Y_{T}^{\prime}$ be the set of vertices $y^{\prime} \in Y^{\prime}$ that are covered by an edge in $M_{T}^{*}$. For each $\iota \in\{1,2, \ldots, N / 2\}$, let $W_{\iota}$ be the set of 4 vertices $x_{\iota}^{\prime}, x_{\iota+N / 2}^{\prime}, y_{\iota}^{\prime}, y_{\iota+N / 2}^{\prime}$. Because $M_{S}^{*}$ and $M_{T}^{*}$ satisfy (1), to prove Lemma 4.1 , it suffices to prove the following statement.

For each $W_{\iota}$, there exists a collection $\mathcal{P}_{\iota}$ of vertex-disjoint directed paths $P$ (some of which may be of length 0 ) in $\Gamma_{N}\left[W_{\iota}\right]$ such that: 
(i') each vertex in $\left(X_{S}^{\prime} \cup X_{T}^{\prime}\right) \cap W_{\iota}$ is the endpoint of exactly one $P \in \mathcal{P}_{\iota}$,

(ii') each $P_{\iota} \in \mathcal{P}_{\iota}$ is either (a) from $X_{S}^{\prime}$ to $X^{\prime \prime}$, (b) from $Y^{\prime \prime}$ to $Y_{T}^{\prime}$, or (c) from $X_{S}^{\prime}$ to $X_{T}^{\prime}$

then $\mathcal{P}=\cup_{\iota} \mathcal{P}_{\iota}$.

The existence of such $\mathcal{P}_{\iota}$ is easy to prove by exhaustive search, since $W_{\iota}$ has only 4 vertices. Indeed, one can easily check that unless either $X_{T}^{\prime} \cap W_{\iota}$ has 2 vertices and $X_{S}^{\prime} \cap W_{\iota}$ none, or $X_{S}^{\prime} \cap W_{\iota}$ has 2 vertices and $X_{T}^{\prime} \cap W_{\iota}$ none, there does exist such a $\mathcal{P}_{\iota}$. But because $M_{S}^{*}$ and $M_{T}^{*}$ satisfy (2), we are assured that neither of these can happen, and so Lemma 4.1 follows.

With Lemma 4.1 in mind, we devote the rest of this section to proving the following proposition.

Proposition 4.2. Let $S$ be any subset of $X$, and let $T$ be any subset of $Y$ where $|S|=|T|$. Then there exist matchings $M_{S}^{*} \subset \Lambda_{X}$ and $M_{T}^{*} \subset \Lambda_{Y}$ with $|S|=|T|$ edges each that satisfy (1) and (2) of Lemma 4.1.

Proof. We first prove Lemma 4.3 stated below, and then use it to establish Proposition 4.2.

Lemma 4.3. Let $S$ and $T$ be as in Proposition 4.2. Then there exist matchings $M_{S}$ and $M_{T}$, and a subset $I$ of $\{1, \ldots, N\}$ that satisfy the following properties.

(1) Each edge in $M_{S}$ is incident with a vertex in $S$, and each edge in $M_{T}$ is incident with a vertex in $T$.

(2) Let $X_{I}^{\prime}$ denote the subset of $X^{\prime}$ of the form $\left\{x_{\iota}^{\prime} \mid \iota \in I\right\}$, and let $Y_{I}^{\prime}$ denote the subset of $Y^{\prime}$ of the form $\left\{y_{\iota}^{\prime} \mid \iota \in I\right\}$. Then $M_{S}$ saturates $X_{I}^{\prime}$ and $M_{T}$ saturates $Y_{I}^{\prime}$.

(3) Let $\alpha$ be the number between 0 and 1 such that $|S|=|T|=\alpha|N|$. If $\alpha$ satisfies $1 / 4 \leq \alpha \leq 1 / 2$, then $|I|$ is at least $(\alpha-1 / 4) N$. If $\alpha$ is larger than $1 / 2$, then $|I|$ is at least $(\alpha-(1-\alpha) / 2) N$.

The proof of Lemma 4.3 uses the following lemma.

Lemma 4.4. Let $\bar{\Lambda}$ be the extended double cover of a 9-regular Ramanujan graph with $N$ vertices, and let $X$ and $Y$ denote its parts, where $|X|=|Y|=N$. Let $S$ 
be a subset of $X$, and let $\alpha$ be such that $|S|=\alpha N$. Then the following hold.

If $\alpha \leq 1 / 4$, then $\left|\mathcal{N}_{\bar{\Lambda}}(S)\right|$ is at least $2|S|$.

If $1 / 4 \leq \alpha \leq 1 / 2$, then $\left|\mathcal{N}_{\bar{\Lambda}}(S)\right|$ is at least $|S|+N / 4$.

If $1 / 2 \leq \alpha$, then $\left|\mathcal{N}_{\bar{\Lambda}}(S)\right|$ is at least $|S|+(1-\alpha) N / 2$.

Proof. Lemma 4.4 is proved using the technique in [Alon et al. 87]. We proceed with the proof. The following lemma is a restatement of Theorem 2.1 in [Alon et al. 87].

Lemma 4.5. Let $G=(V, E)$ be a d-regular graph on $N$ vertices, and suppose that the second largest eigenvalue of the adjacency matrix of $G$ is at most $\lambda$. Define $a=\frac{d-\lambda}{2 d}, b=\frac{1+2 a}{4 a}$. Let $S \subset V$ be a set of vertices of $G$, and let $W=\mathcal{N}_{G}(S)-S$ be the set of all neighbors of $S$ that lie outside $S$. Put $|S|=\alpha N$ and $|W|=w N$. Then

$$
w^{2}-2(1-2 \alpha-b) w-4 \alpha(1-\alpha) \geq 0 .
$$

Note that if $G$ is as in Lemma 4.5, and $H$ is the extended double cover of $G$, then for every set of vertices $S \subset X$ satisfying $|S|=\alpha N$ and $\left|\mathcal{N}_{H}(S)\right|=$ $(\alpha+w) N$, the inequality (4.1) holds. In Lemma 4.4, we start with a 9-regular Ramanujan graph on $N$ vertices. As it is Ramanujan, its second largest eigenvalue is at most $2 \sqrt{8}$. Therefore, in the notation of Lemma 4.5, $a=\frac{9-2 \sqrt{8}}{18}=0.18573$ and $b=\frac{1+2 a}{4 a}=1.846038$. Let $S$ and $W$ be as in Lemma 4.5; suppose $|S|=\alpha N$ and $|W|=w N$. Substituting in (4.1), we get

$$
w^{2}+(1.6920754+4 \alpha) w-4 \alpha(1-\alpha) \geq 0 .
$$

Therefore, in order to complete the proof of Lemma 4.4, it suffices to prove the following.

Lemma 4.6. Let $\alpha$ and $w$ be two reals in $[0,1]$ and suppose (4.2) holds. Then:

(i) If $\alpha \leq 1 / 4$, then $w \geq \alpha$.

(ii) If $1 / 4 \leq \alpha \leq 1 / 2$, then $w \geq 1 / 4$.

(iii) If $\alpha \geq 1 / 2$, then $w \geq \frac{1-\alpha}{2}$.

Proof. For every fixed $\alpha \in[0,1]$ the left-hand side of $(4.2)$, which is

$$
f(w)=w^{2}+(1.6920754+4 \alpha) w-4 \alpha(1-\alpha),
$$


is a strictly increasing function of $w$ for $w \in[0,1]$. Therefore, it suffices to prove the following:

(i') If $0 \leq \alpha \leq 1 / 4$, then

$$
f(\alpha)=\alpha^{2}+(1.6920754+4 \alpha) \alpha-4 \alpha(1-\alpha) \leq 0 .
$$

(ii') If $1 / 4 \leq \alpha \leq 1 / 2$, then

$$
f(1 / 4)=1 / 16+(1.6920754+4 \alpha) 1 / 4-4 \cdot \alpha(1-\alpha) \leq 0 .
$$

(iii') If $1 / 2 \leq \alpha \leq 1$, then

$$
f\left(\frac{1-\alpha}{2}\right)=\left(\frac{1-\alpha}{2}\right)^{2}+(1.6920754+4 \alpha) \frac{1-\alpha}{2}-4 \alpha(1-\alpha) \leq 0 .
$$

These inequalities can be checked routinely. The left-hand side of (4.3) is nonpositive for all $\alpha \in[0,0.2564]$ (and hence for all $\alpha \in[0,1 / 4]$ ); the left-hand side of (4.4) is nonpositive for all $\alpha \in[0.236314,0.51368]$ (and hence for all $\alpha \in[1 / 4,1 / 2])$; and the left-hand side of (4.5) is nonpositive for all $\alpha \in[0.4872,1]$ (and hence for all $\alpha \in[1 / 2,1]$ ). This completes the proof, and hence implies the assertion of Lemma 4.4 as well.

We now use Lemma 4.4 together with Menger's Theorem to prove the existence of $I, M_{S}$ and $M_{T}$ that satisfy (1)-(3) of Lemma 4.3, and then this lemma will follow. Let $U^{\prime}=\left\{u_{1}^{\prime}, \ldots, u_{N}^{\prime}\right\}$ and $V^{\prime}=\left\{v_{1}^{\prime}, \ldots, v_{N}^{\prime}\right\}$ be disjoint sets, each of $N$ vertices. Let $G^{\prime}$ be the following graph on $S \cup U^{\prime} \cup V^{\prime} \cup T$. Every edge in $G^{\prime}$ is either directed from $S$ to $U^{\prime}$, or from $U^{\prime}$ to $V^{\prime}$, or from $V^{\prime}$ to $T$.

- $G\left[S \cup X^{\prime}\right]$ is isomorphic to $\Lambda_{X}\left[S \cup X^{\prime}\right] ;\left(x, u_{\iota}^{\prime}\right)$ in an edge in $G^{\prime}$ if and only if $\left(x, x_{\iota}^{\prime}\right)$ is in $\Gamma_{N}($ and $x \in S)$.

- $G^{\prime}\left[U^{\prime} \cup V^{\prime}\right]$ is a matching; $\left(u_{\iota}^{\prime}, v_{\iota^{\prime}}^{\prime}\right)$ is an edge in $G^{\prime}$ if and only if $\iota=\iota^{\prime}$.

- $G\left[Y^{\prime} \cup T\right]$ is isomorphic to $\Lambda_{Y}\left[Y^{\prime} \cup T\right] ;\left(v_{\iota}^{\prime}, y\right)$ is an edge in $G^{\prime}$ if and only if $\left(y_{\iota}^{\prime}, y\right)$ is an edge in $\Gamma_{N}$ (and $\left.y \in T\right)$.

Note that the maximum possible number of vertex-disjoint paths from $S$ to $T$ is the maximum possible cardinality of $I$ in Lemma 4.3. By Menger's Theorem, we have the following fact:

(M) The maximum possible size of $I$ is equal to the minimum possible cardinality of a set of vertices $C$ that separates $S$ and $T$ in $G^{\prime}$. 
Let $C$ be a minimum size separating set, and let $a, b, c, d \in[0,1]$ satisfy $|C \cap S|$ $=a N ;\left|C \cap U^{\prime}\right|=b N ;\left|C \cap V^{\prime}\right|=c N$; and $|C \cap T|=d N$. Thus, $|C|=(a+b+c+d) N$.

Consider two possible cases.

Case I: $1 / 4 \leq \alpha \leq 1 / 2$. Then we may assume that both $a$ and $d$ are no larger than $\alpha-1 / 4$, or equivalently, that $S \backslash C$ and $T \backslash C$ have at least $N / 4$ vertices each, or we are done by (M). By Lemma 4.4, there are at least $|S|-a N+N / 4$ $=(\alpha-a+1 / 4) N$ vertices in $U^{\prime}$ that are adjacent to at least one vertex in $S \backslash C$, and at least $|S|-d+N / 4=(\alpha-d+1 / 4) N$ vertices in $V^{\prime}$ that are adjacent to at least one vertex in $T \backslash C$. Thus, there are at least $(2 \alpha-d-a-1 / 2) N$ indices $\iota$ such that both $u_{\iota}^{\prime}$ is adjacent to a vertex in $S \backslash C$, and $v_{\iota}^{\prime}$ is adjacent to a vertex in $T \backslash C$. Therefore, $\left|C \cap\left(U^{\prime} \cup V^{\prime}\right)\right|$ must be at least $(2 \alpha-a-d-1 / 2) N$, which implies that $|C|$ must be at least $(2 \alpha-1 / 2) N$. Thus, by (M), the assertion of Lemma 4.3 follows.

Case 2: $1 / 2 \leq \alpha$. Then we may assume by (M) that both $\alpha-a$ and $\alpha-d$ are at least $(1-\alpha) / 2$, or equivalently, that $S \backslash C$ and $T \backslash C$ both have at least $(1-\alpha) / 2$ vertices. By Lemma 4.4 , there are at least $|S|-a N+(1-\alpha) N / 2=$ $(\alpha-a+(1-\alpha) / 2) N$ vertices in $U^{\prime}$ that are adjacent to at least one vertex in $S \backslash C$, and at least $(\alpha-d+(1-\alpha) / 2) N$ vertices in $V^{\prime}$ that are adjacent to at least one vertex in $T \backslash C$. Hence, $C \cap\left(U^{\prime} \cup U^{\prime}\right)$ must be at least $(\alpha-a-d) N$, which implies that $|C|$ must be at least $\alpha N$. The assertion of Lemma 4.3 follows.

We now use Lemma 4.3 to complete the proof of Proposition 4.2. We prove that there exist matchings $M_{S}^{*} \subset \Lambda_{X}$ and $M_{T}^{*} \subset \Lambda_{Y}$ with $|S|=|T|$ edges each that satisfy (1) of Lemma 4.1, and that satisfy (A) and (B), stated below.

(A) $M_{S}^{*}$ saturates $X_{I}^{\prime}$, and $M_{T}^{*}$ saturates $Y_{I}^{\prime}$.

(B) Let $\iota$ be an arbitrary integer in $\{1, \ldots, N / 2\}$ such that neither $\iota$ nor $\iota+N / 2$ is in $I$. Then $M_{S}^{*}$ covers at most one of $x_{\iota}^{\prime}, x_{\iota+N / 2}^{\prime}$, and $M_{T}^{*}$ covers at most one of $y_{\iota}^{\prime}, y_{\iota+N / 2}^{\prime}$.

Then $M_{S}^{*}$ and $M_{T}^{*}$ will satisfy (2) of Lemma 4.1 as well, and Proposition 4.2 will follow.

Now let $M_{S}, M_{T}$, and $I$ satisfy (1)-(3) of Lemma 4.3, and let $X_{I}^{\prime}$ and $Y_{I}^{\prime}$ be as in (2) of Lemma 4.3. To show that there exist $M_{S}^{*}$ and $M_{T}^{*}$ as needed, let $G_{S}^{\prime}$ be the graph $\Lambda_{X}\left[S \cup X^{\prime}\right]$. Next, let $G_{S}$ be the graph formed from $G$ by identifying $x_{\iota}^{\prime}$ with $x_{\iota+N / 2}^{\prime}$ if and only if neither the integer $\iota$ nor $\iota+N / 2$ is in $I$. Construct $G_{T}$ in an analogous fashion. Thus, matchings in $G_{S}$ and $G_{T}$ that saturate both $S$ and $X_{I}^{\prime}$ simultaneously, and $T$ and $Y_{I}^{\prime}$ simultaneously, respectively, correspond to matchings in $\Lambda_{X}$ and $\Lambda_{Y}$ with 
$|S|$ edges each that satisfy both (1) of Lemma 4.1 and (A) and (B), and thus, (2) of Lemma 4.1. Hence, to finish the proof of Proposition 4.2, it suffices to prove that:

(C) There exist matchings in $G_{S}$ and $G_{T}$ that saturate both $S$ and $X_{I}^{\prime}$ simultaneously, and $T$ and $Y_{I}^{\prime}$ simultaneously, respectively.

This is done next. We first show a statement that seems a priori weaker than $(\mathrm{C})$, namely:

(D) There exist matchings in $G_{S}$ and $G_{T}$ that saturate $S$ and $T$, respectively, and there exist (a possibly different set of) matchings in $G_{S}$ and $G_{T}$ that saturate $X_{I}^{\prime}$ and $Y_{I}^{\prime}$, respectively.

We claim that (D) implies (C). Indeed, suppose $M_{1}$ is a matching in $\hat{\Lambda}_{X}$ that saturates $S$ and $M_{2}$ a matching that saturates $X_{I}^{\prime}$. Let $C$ be a component of the union $M_{1} \cup M_{2}$, and let $P$ be the set of edges of $M_{1} \cup M_{2}$ incident to a vertex in $C$. It is not difficult to check that there is a subset $S_{C}$ of edges of $P$ that is a matching, and that saturates $C \cap\left(X_{I}^{\prime} \cup S\right)$. The union $\cup_{C} S_{C}$, where $C$ ranges over the components of $M_{1} \cup M_{2}$, is a matching that covers every vertex of $X_{I}^{\prime}$ and $S$. A similar statement holds in $G_{T}$. Thus, (D) implies (C).

Having shown that (D) implies (C), we now prove (D). Since $M_{S}, M_{T}$, and $I$ satisfy (1) and (2) of Lemma 4.3, there exist matchings in $G_{S}$ and $G_{T}$ that saturate $X_{I}^{\prime}$ and $Y_{I}^{\prime}$, respectively. Therefore, to prove (D), all we need to show now is that there exist matchings in $G_{S}$ and $G_{T}$ that saturate $S$ and $T$, respectively. We do this next, using the fact that $I$ satisfies (3) of Lemma 4.3 as well. By Hall's Theorem, there exist such matchings if, for each subset $S_{0}$ of $S$, and each subset $T_{0}$ of $T,\left|\mathcal{N}_{G_{S}}\left(S_{0}\right)\right| \geq\left|S_{0}\right|$, and $\left|\mathcal{N}_{G_{T}}\left(T_{0}\right)\right| \geq\left|T_{0}\right|$. But we note that

$$
\left|\mathcal{N}_{G_{S}}\left(S_{0}\right)\right| \geq\left|\mathcal{N}_{G_{S}}\left(S_{0}\right) \cap X_{I}^{\prime}\right|+\left|\mathcal{N}_{G_{S}}\left(S_{0}\right) \cap\left(X^{\prime} \backslash X_{I}^{\prime}\right)\right| / 2 .
$$

We now consider 2 cases.

Case I: $\alpha \leq 1 / 2$, where $|S|=\alpha N$, as before. The case when $\left|S_{0}\right|$ is no larger than $N / 4$ can be handled easily using Lemma 4.4. If $\left|S_{0}\right|$ is at least $N / 4$, then by Lemma 4.4, $\left|\mathcal{N}_{G_{S}}\left(S_{0}\right)\right|$ is at least $\left|S_{0}\right|+N / 4$. Also, $|I|$ is at least $(\alpha-1 / 4) N$ because $I$ satisfies (3) of Lemma 4.3. So $\left|\mathcal{N}_{G_{S}}\left(S_{0}\right) \cap X_{I}^{\prime}\right| \geq\left|S_{0}\right|-N / 4$, because at most $N / 4$ vertices in $S$ are not matched by $M_{S}$ to a vertex in $X_{I}^{\prime}$ (as $M_{S}$ and $I$ satisfy (1) and (2) of Lemma 4.3). Together, these imply that $\left|\mathcal{N}_{G_{S}}\left(S_{0}\right)\right| \geq$ $\left|S_{0}\right|$. Similarly, $\left|\mathcal{N}_{G_{T}}\left(T_{0}\right)\right| \geq\left|T_{0}\right|$. This implies (D) for the case $\alpha \leq 1 / 2$.

Case 2: $\alpha \geq 1 / 2$. Then we may assume that $\left|S_{0}\right| \geq(1-\alpha)|N| / 2$. By Lemma 4.4, we see that $\left|\mathcal{N}_{G_{S}}\left(S_{0}\right)\right|$ is at least $\left|S_{0}\right|+(1-\alpha) N / 2$. Also, $|I|$ is at least 
$(\alpha-(1-\alpha) / 2) N$ because $I$ satisfies (3) of Lemma 4.3. So $\left|\mathcal{N}_{G_{S}}\left(S_{0}\right) \cap X_{I}^{\prime}\right| \geq$ $\left|S_{0}\right|-(1-\alpha) N / 2$ because at most $(1-\alpha) N / 2$ vertices in $S$ are not matched by $M_{S}$ to a vertex in $X_{I}^{\prime}$ (because $M_{S}$ and $I$ satisfy (1) and (2) of Lemma 4.3). Together, these imply that $\left|\mathcal{N}_{G_{S}}\left(S_{0}\right)\right| \geq\left|S_{0}\right|$. Similarly, $\left|\mathcal{N}_{G_{T}}\left(T_{0}\right)\right| \geq\left|T_{0}\right|$. This implies (D) for the case when $\alpha \geq 1 / 2$ as well, and hence (D) follows.

As we have shown, (D) implies (C), which implies Proposition 4.2 by the discussion preceding the statement of $(\mathrm{C})$.

Proposition 4.2 and Lemma 4.1 imply Theorem 3.1.

\section{Acknowledgments}

We would like to thank Alex Lubotzky for helpful discussions. N. Alon's research was supported in part by a USA Israeli BSF grant, by a grant from the Israel Science Foundation, and by the Hermann Minkowski Minerva Center for Geometry at Tel Aviv University. M. Capalbo's research was supported by NSF grant CCR98210-58.

\section{Note Added in Proof}

The construction described here can be slightly modified to yield an explicit construction of an infinite family of $N$-superconcentrators of density 40 . Since the basic idea and analysis are similar to our treatment here, but the details are significantly more complicated, we refer the interested reader to http://www.dimacs.rutgers. $\mathrm{edu} /{ }^{\sim}$ mcapalbo for the detailed proof, and only sketch here the modified construction. As in the original construction, the modified one starts with 4 pairwise disjoint sets of vertices $X, X^{\prime}, Y, Y^{\prime}$, each of cardinality $N$. The edges between $X$ and $X^{\prime}$, and those between $Y$ and $Y^{\prime}$, are double covers of the 9-regular Ramanujan graph described explicitly in Section 2. Note that these are double covers, and not extended double covers, that is, we do not include the edges $x_{\iota} x_{\iota}^{\prime}$ and $y_{\iota} y_{\iota}^{\prime}$. In addition, we fix a generator $\pi$ of our Ramanujan graph, and recall that its order is 2 . The vertices of $X, X^{\prime}$ are numbered in such a way that for every $\iota$ between 1 and $N / 2$, the generator $\pi$ maps $x_{\iota}$ to $x_{\iota+N / 2}$ and $x_{\iota}^{\prime}$ to $x_{\iota+N / 2}^{\prime}$, and similarly for the vertices $y_{i}, y_{i}^{\prime}$. The rest of the construction is identical to the description given in Section 3. The number of edges $f(N)$ of the graph with $N$ inputs and $N$ outputs constructed this way clearly satisfies $f(N)=(2 \cdot 9+2) N+f(N / 2)$, implying that $f(N)=40 N+O(1)$. The detailed proof that this graph is indeed an $N$-superconcentrator can be found at http://www.dimacs.rutgers.edu/ ${ }^{\text {mcapalbo. }}$

\section{References}

[Alon et al. 87] N. Alon, Z. Galil, and V.D. Milman. "Better Expanders and Superconcentrators." J. Algorithms 8 (1987), 337-347. 
[Alon and Milman 85] N. Alon and V.D. Milman. " $\lambda_{1}$, Isoperimetric Inequalities for Graphs and Superconcentrators." J. Combinatorial Theory B 38 (1985), 77-88.

[Buck 86] M. Buck. "Expanders and Diffusers. SIAM J. Algebraic and Disc. Meth. 7 (1986), 282-304.

[Chung 78] F. R. K. Chung. "On Concentrators, Superconcentrators, Generalizers and Nonblocking Networks." Bell System Tech. J. 58 (1978), 1765-1777.

[Gabber and Galil 79] O. Gabber and Z. Galil. "Explicit Construction of Linear-Sized Superconcentrators." FOCS (1979), 364-370.

[Jimbo and Maruoka 87] A. Jimbo and A. Maruoka. "Expanders Obtained from Affine Transformations." Combinatorica 7 (1987), 343-345.

[Lev and Valiant 83] G. Lev and L. G. Valiant. "Size Bounds for Superconcentrators." Theoret. Comput. Sci. 22 (1983), 233-251.

[Lubotzky 02] A. Lubotzky. Personal communication, 2002.

[Lubotzky et al. 88] A. Lubotzky, R. Phillips, and P. Sarnak. "Ramanujan Graphs. Combinatorica 8 (1988), 261-277.

[Morgenstern 94] M. Morgenstern. "Existence and Explicit Construction of $\mathrm{q}+1$ Regular Ramanujan Graphs for Every Prime Power q." J. Combinatorial Theory B 62 (1994), 44-62.

[Pippenger 77] N. Pippenger. "Superconcentrators." SIAM J. Comput. 6 (1977), 298304.

[Valiant 76] L. G. Valiant. "Graph Theoretic Properties in Computational Complexity." J. Comput. System Sci. 13 (1976), 278-285.

N. Alon, Department of Mathematics, Tel Aviv University, Tel Aviv 69978, Israel (nogaa@post.tau.ac.il)

M. Capalbo, Institute for Advanced Study, Princeton, NJ 08540 (mrc@ias.edu)

Received March 10, 2003; accepted March 26, 2003. 
